\title{
Some trends on how one can learn from and mimic nature in order to design better biomaterials
}

It is our great privilege as Director and one of the main organizers of the NATO Advanced Study Institute (ASI) on "Learning from Nature How to Design New Implantable Biomaterials: From Biomineralization Fundamentals to Biomimetic Materials and Processing Routes", held from the 13th to the 24th of October 2003 in Alvor, Algarve, Portugal, to introduce you to a selection of papers resulting from the original contributions presented by the ASI students.

It is rather typical that an ASI results on a state of the art book composed by invited chapters prepared by the ASI faculty. This was also the case for this NATO-ASI and a wonderful book has already been published by Kluwer. These books are of course very useful research and education tools. This one resulted very well and, if you are interested in the topic, you should really look for it. Nevertheless, in NATO courses, the ASI students also have the opportunity to present their original works as posters or in some cases as short oral presentations. During the course and when looking at the quality of some of the works, we have decided to propose to Prof. Paul Calvert (also a Lecturer at the ASI) the preparation of a special issue of Materials Science and Engineering C: Biomimetic and Supramolecular Systems, containing a selection of the best works presented at the course. He and Elsevier welcomed the idea, and it was decided we should both act as Guest Editors. After a careful reviewing process, we ended up with the papers contained in this special issue.

It is our deepest belief that the development of materials for any replacement or regeneration application should be based on the thorough understanding of the structure to be substituted. This is true in many fields, but particularly exigent in substitution and regeneration medicine. The demands upon the material properties largely depend on the site of application and the function it has to restore. Ideally, a replacement material should mimic the living tissue from a mechanical, chemical, biological and functional point of view. Of course, this is much easier to write down than to implement in clinical practice.

Mineralized tissues such as bones, tooth and shells have attracted, in the last few years, considerable interest as natural anisotropic composite structures with adequate mechanical properties. In fact, Nature is and will continue to be the best material scientist ever. Who better than Nature can design complex structures and control the intricate phenomena (processing routes) that lead to the final shape and structure (from the macro to the nano level) of living creatures? Who can combine biological and physicochemical mechanisms in such a way that can build ideal structure-properties relationships? Who else than Nature can really design smart structural components that respond in-situ to exterior stimulus, being able of adapting constantly their microstructure and correspondent properties? In the described philosophy line, mineralized tissues and biomineralization processes are ideal examples to learnfrom for the materials scientist of the future.

In the last few years, it has been an increasing interest in the understanding how organism produce their minerals, which could help us on the development of new and superior materials. The evident hypothesis has been to try to mimic some of the imaginative strategies selected by Nature to continuously improve the performance of natural materials for the required necessities, as carried out throughout the evolutionary process. Mineralized materials found in Nature exhibit often complex hierarchical composite structures. Even apparently simpler organisations, such as the "brick-wall" structure of nacre, are very difficult to be reproduced synthetically, as the underlying formation mechanisms are biologically controlled from the nanometric to the macroscopic scales, involving biomacromolecules in both nucleation and the growth processes. Nevertheless, the understanding of the general processes involved in biomineralization, and the establishment of structure/properties relationships in the resulting materials, have inspired the development of new concepts both for the design and the processing of distinct parts and materials. This is true in different materials science and engineering fields, such as in electronics and optics, and for instances in the fabrication of laminated ceramics or biomimetic coatings. The basic knowledge on biomineralization fundamentals is particularly relevant in the biomedical field, and it might help on the development of materials that will interact well with 
mineralized tissues or, for example, will allow tailoring the properties of composite particles aimed at being used in controlled release of drugs or other bioactive molecules.

Typically, the main characteristics of the route by which the mineralized hard tissues are formed is that the organic matrix is laid down first and the inorganic reinforcing phase grows within this organic matrix/template. Bone, tooth, lobster and crabs exoskeletons, oyster shells, coral, ivory, pearls, sea urchin spines, cuttlefish bone, are just a few of the wide variety of biomineralized materials engineered by living creatures. Many of these biological structural materials consist on inorganic minerals combined with organic polymers. The study of these structures has generated a growing awareness that the adaptation of biological processes may lead to significant advances in the controlled fabrication of novel and better-engineered smart materials. To date, neither the elegance of the biomineral assembly mechanisms nor the rather complex composite microarchitectures could be duplicated by nonbiological methods. This is true in spite of the fact that substantial progress has been made in understanding how biomineralization occurs. However, most of this knowledge is yet to be used on relevant industrial applications, namely on the design of appropriate biomimetic routes that will lead to the development of a new generation of implantable biomaterials.

Biomimetics is an immerging field of science that includes the study of how Nature designs, processes and assembles/disassembles molecular building blocks to fabricate high performance mineral-polymer composites (e.g., mollusc shells, bone, tooth) and/or soft materials (e.g., skin, cartilage, tendons), and then applies these designs and processes to engineer new molecules and materials with unique properties. Studies can focus on: the development of methods to reveal the mechanisms through which organic assemblies such as proteins/peptides can determine the biomineral structure of tooth and bone; the determination of tooth and bone hierarchical structure; deciphering of biological basis of biomineralization using paradigms from marine invertebrates; understanding fundamentals of dental enamel proteins self-assembly; the design of a new generation of tooth and bone structure materials based on lessons from the marine mussel and spider silk proteins; and the development of "mineral delivery vehicles" based on phospholipids self-assembly for the repair or re-mineralization of enamel, dentin as well as bone defects. For instances, bone formation is a particularly complex process, on which hydroxylapatite precipitation seems to be associated, initially, with matrix vesicles and subsequently with collagen fibres. Very small crystals are formed, parallel to one another, under the influence of the collagen fibrils structure, as a result of the composition of the inorganic salts present in the body fluids. Bone is synthesized as a complex composite and the organization and interfacial chemistry of the components are optimized for functional use by means of cell-mediated processes.
Many research groups have been active on developing strategies to pre-mineralize or induce a bioactive character in the surface of compact or porous materials aimed to be used on bone replacement or regeneration applications. In fact, in the last few years, it is becoming well established that the essential requirement for an artificial material to exhibit a bone-bonding behaviour is the formation, on its surface, of a calcium phosphate (Ca-P) similar to bone apatite, being well known that the presence of a calciumphosphate coating will promote the fixation of the device onto bone. It has been reported by many authors that the presence of an apatite-like layer on the surface of an orthopaedic biomaterial is considered as a positive biological response from the host tissues. However, one should never forget that the same understanding of the basis of biomineralization may be useful in the opposite sense, that is when one aims to develop biomaterials that should prevent calcification, such as devices for cardiovascular applications or to be used in tissue engineering of cartilage or other soft-tissues; in this case, chemical strategies could be employed to inhibit such mineralization process.

We have decided to organize a NATO Advanced Study Institute on "Learning from Nature How to Design New Implantable Biomaterials: From Biomineralization Fundamentals to Biomimetic Materials and Processing Routes", when we realized that there was a clear need for a course that would address all the above referred to topics. In fact, it was our deep believe that there was a necessity for a course that addressed, in an integrated way, topics that go from understanding biomineralization processes of different mineralized tissues (that means: not only bone, tooth, etc.) to the use of that science to engineer new biomimetic processes and materials. In fact, only an understanding of the relevant fundamentals and a simultaneous application oriented view will lead to the design of new biomimetic materials and processing routes (including production of biomimetic coatings). However, the biomineralization and biomaterials research communities have not been working side by side in the past few years. So we thought that an ASI could be a complementary tool as it seemed to us to be the best forum to educate and brainstorming on this area of such strategic importance. The present collection of papers, together with the already published book, are what will remain as a result of such unique kind of meeting. Furthermore, they will be available to everybody that could not attend the meeting.

The papers of this special issue of Materials Science and Engineering C: Biomimetic and Supramolecular Systems reflect the main scientific areas that were addressed in the NATO-ASI. As expected in such a multidisciplinary area, a variety of fields were covered, from fundamental aspects on biomineralization up to practical concepts to be implemented in biomedical applications using biomimetic approaches.

We have the privilege to open this special issue with a review paper by Prof. Tadashi Kokubo, on the essential 
principles related to the development of bioactive materials. Then, the order of the selected papers is organized as follows:

- Characterisation of natural mineralized structures: studies include the analysis of proteins existing in shells, directly affecting the calcification of organisms, the investigation of both structure and properties (including the mechanical/viscoelastic behaviour) of mineralized biological materials, such as sea shells, bone or petrified wood, and further insights on the crystalline structure of apatite in bone.

- Biomineralization and biomimetic strategies: methods for controlled calcification are presented including the use of new sources of carbon dioxide for precipitation of calcium carbonate, and the study of the effect of charge density in the growth of this mineral onto amphiphilic monolayers. Another study also investigates the incorporation of proteins within biomimetic calcium phosphate coatings.

- Processing and modification of biomaterials: novel methodologies are presented to process bulk (porous apatite scaffolds) bioactive biomaterials or induce a bioactive behaviour in biomaterials' surface (silica based coatings). Polymeric-based materials are also processed into 3D-porous structures, aimed at being used as support for tissue engineering of bone or cartilage.

- Controlled release from mineral structures: some authors were interested in analysing the effect of mineralization, or composition/structure relationships in composites, on the release features of entrapped molecules.

- Biological response to bioactive systems: the two last papers include the study of cell adhesion and proliferation in bioactive polymeric matrix composites.

Finally, we must say that, as most of you know, nobody can organize a course without the help of hard working people and support from several institutions. We would of course first of all like to thank the NATO Scientific Division for their support that made possible the course. We would like to acknowledge the many contributions of our co-director and our friend Steve Weiner. He was a great support whenever we needed it. The members of the scientific committee and several of the lectures made a lot of useful suggestions. We wish to thank all the invited speakers that made the course possible. But the course, and the program, were also made by the ASI students and their wonderful contributions. The best of these contributions lead to this special issue. The many reviewers involved on the refereeing process are also gratefully acknowledged. But we are especially grateful to our group, our colleagues, our secretary, post-docs and $\mathrm{PhD}$ students. The outcome of this ASI was mainly the result of their hard work, dedication and ambition. They have put a great number of hours on this enterprise and realized that this was an important organization for all of us. We cannot refer all the names herein, but if you find one of the members of the 3B's Research Group-Biomaterials, Biodegradables and Biomimetics in one of the meetings you attend, please just speak with her/him and you will see how fortunate we are for being able to advise such a wonderful group of young and bright researchers.

I hope you all enjoy this special issue as much as we have enjoyed preparing it.

\section{João F. Mano \\ Rui L. Reis* \\ (Guest Editors)}

3Bs Research Group-Biomaterials, Biodegradables and Biomimetics, University of Minho, Campus de Gualtar, 4710-057 Braga, Portugal Department of Polymer Engineering, University of Minho, Campus de Azurém, 4800-058 Guimarães, Portugal E-mail addresses: jmano@dep.uminho.pt (J.F. Mano), rgreis@dep.uminho.pt (R.L. Reis). $U R L$ : www.dep.uminho.pt/3bs. *Corresponding author. 